CRYSTALLOGRAPHIC COMMUNICATIONS

ISSN 2056-9890

Received 22 November 2018

Accepted 28 November 2018

Edited by W. T. A. Harrison, University of Aberdeen, Scotland

\# Thomson Reuters ResearcherID: F-88162012.

$\S$ Thomson Reuters ResearcherID: A-55252009.

Keywords: crystal structure; polymorph; hydrogen-bond; 3-methoxybenzoic acid.

CCDC reference: 1448794

Supporting information: this article has supporting information at journals.iucr.org/e

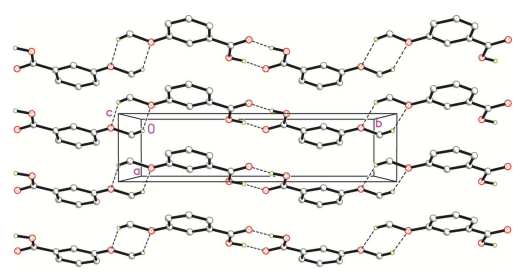

OPEN $\odot$ ACCESS

\section{Crystal structure of a second monoclinic polymorph of 3-methoxybenzoic acid with $Z^{\prime}=1$}

\author{
Tze Shyang Chia, ${ }^{\mathrm{a}}$ \# Huey Chong Kwong, ${ }^{\mathrm{b}}$ Qin Ai Wong, ${ }^{\mathrm{a}}$ Ching Kheng Quah ${ }^{\mathrm{a}} \S$ and \\ Md. Azharul Arafath ${ }^{\mathrm{C} *}$

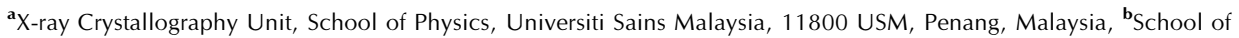
Chemical Sciences, Universiti Sains Malaysia, 11800 USM, Penang, Malaysia, and ' ${ }^{\mathbf{D}}$ Department of Chemistry, Shahjalal University of Science and Technology, Sylhet, 3114, Bangladesh. *Correspondence e-mail:
\end{abstract} \\ arafath_sustche90@yahoo.com
}

A new polymorphic form of the title compound, $\mathrm{C}_{8} \mathrm{H}_{8} \mathrm{O}_{3}$, is described in the centrosymmetric monoclinic space group $P 2_{1} / c$ with $Z^{\prime}=1$ as compared to the first polymorph, which crystallizes with two conformers $\left(Z^{\prime}=2\right)$ in the asymmetric unit in the same space group. In the crystal of the second polymorph, inversion dimers linked by $\mathrm{O}-\mathrm{H} \cdots \mathrm{O}$ hydrogen bonds occur and these are linked into zigzag chains, propagating along the $b$-axis direction by $\mathrm{C}-$ $\mathrm{H}$. . O links. The crystal structure also features a weak $\pi-\pi$ interaction, with a centroid-to-centroid distance of 3.8018 (6) $\AA$. The second polymorph of the title compound is less stable than the reported first polymorph, as indicated by its smaller calculated lattice energy.

\section{Chemical context}

Methoxybenzoic acid, also called anisic acid, consists of three isomers with molecular formula $\mathrm{C}_{8} \mathrm{H}_{8} \mathrm{O}_{3}$ : the crystal structures of 2- and 4-methoxybenzoic acids with $Z^{\prime}=1$ have been reported (Parvez, 1987; Etter et al., 1988; Bryan, 1967; Colapietro \& Domenicano, 1978; Fausto et al., 1997; Hathwar et al., 2011) and polymorphism has not been observed for these two isomers in the Cambridge Structural Database (CSD) (Version 5.39, last update August 2018; Groom et al., 2016) to date. In this article, we report a second polymorphic form (I $\beta$ ) of 3-methoxybenzoic acid with $Z^{\prime}=1$ and compare its properties with those of the previously reported first polymorphic form (I $\alpha$ ). Polymorph $\mathrm{I} \alpha$ crystallizes in the monoclinic space group $P 2_{1} / n$ with $a=13.8034$ (17) $\AA, b=5.0275$ (5) $\AA, c=$ 21.446 (3) $\AA$ and $\beta=99.320$ (13) (Raffo et al., 2014; refcode EFINEO). The asymmetric unit of I $\alpha$ consists of two molecules with different conformations $\left(Z^{\prime}=2\right)$, which are connected into a homodimer through strong $\mathrm{O}-\mathrm{H} \cdots \mathrm{O}$ hydrogen bonds. As described below, these two conformers ( $A$ and $B$ ) differ in the orientation of the methoxy group and its relative position from the $-\mathrm{OH}$ group. DFT calculations suggest that the $A$ conformer of $\mathrm{I} \alpha$ is more energetically stable than the $B$ conformer (Pereira Silva et al., 2015).<smiles>COc1cccc(C(=O)O)c1</smiles>

conformer $A$<smiles>COc1cccc(C(=O)O)c1</smiles>

conformer $B$ 


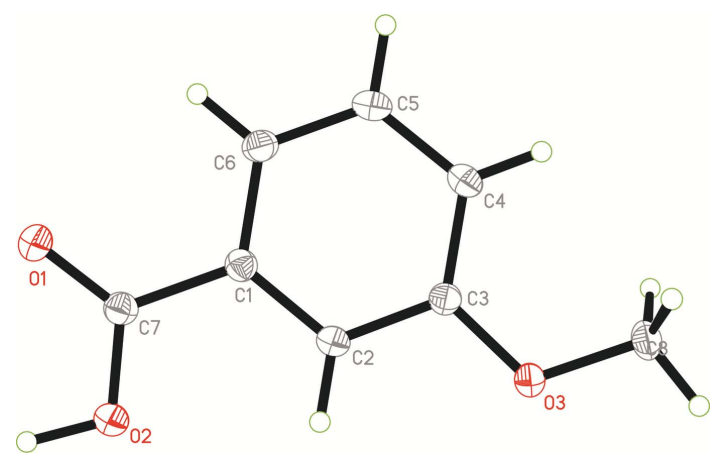

Figure 1

The molecular structure of $\mathrm{I} \beta$ with $50 \%$ probability displacement ellipsoids.

\section{Structural commentary}

The asymmetric unit of $\mathrm{I} \beta$ (Fig. 1) consists of a unique 3-methoxybenzoic acid molecule $\left(Z^{\prime}=1\right)$. The molecule is almost planar with a maximum deviation of 0.107 (1) $\AA$ at atom $\mathrm{O} 1$. The molecules of $\mathrm{I} \beta$ adopt a similar conformation (overlay r.m.s.d. $=0.052 \AA$ ) as compared to the conformer $A$ of $\mathrm{I} \alpha$ (Raffo et al., 2014). The carboxyl group (O1/O2/C7/H1O2) of $\mathrm{I} \beta$ is close to coplanar with the attached phenyl ring $(\mathrm{Cl}-$ C6) as indicated by the dihedral angle of $5.6(7)^{\circ}$. The $\mathrm{C} 8-$ $\mathrm{O} 3-\mathrm{C} 3-\mathrm{C} 2$ torsion angle of $\mathrm{I} \beta$ is $-176.63(7)^{\circ}$ as compared to $-176.75(11)$ and $-1.4(2)^{\circ}$ for conformers $A$ and $B$, respectively, of $\mathrm{I} \alpha$.

\section{Supramolecular features}

In the crystal of $\mathrm{I} \beta$, two inversion-related molecules are joined into a homodimer with an $R_{2}^{2}(8)$ graph-set motif via strong pairwise $\mathrm{O}-\mathrm{H} \cdots \mathrm{O}$ hydrogen bonds (Fig. 2, Table 1). The homodimers are linked by weak $\mathrm{C}-\mathrm{H} \cdots \mathrm{O}$ hydrogen bonds between two methoxy groups into zigzag chains with $R_{2}^{2}(6)$ graph-set motifs, which propagate along the $b$-axis direction. The [010] chains are stacked along the $a$ axis into corrugated sheets parallel to the $a b$ plane via weak $\pi-\pi$ interactions with a

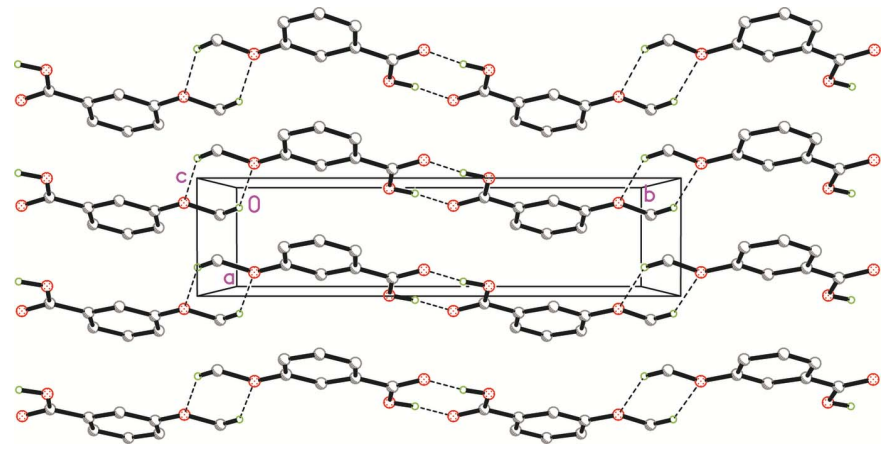

Figure 2

Partial crystal packing of I $\beta$. Dashed lines represent the hydrogen-bonds. Hydrogen atoms not involved in hydrogen bonding are omitted for clarity.
Table 1

Hydrogen-bond geometry $\left(\AA,^{\circ}\right)$.

\begin{tabular}{lllll}
\hline$D-\mathrm{H} \cdots A$ & $D-\mathrm{H}$ & $\mathrm{H} \cdots A$ & $D \cdots A$ & $D-\mathrm{H} \cdots A$ \\
\hline $\mathrm{O} 2-\mathrm{H} 1 O 2 \cdots \mathrm{O} 1^{\mathrm{i}}$ & $1.008(19)$ & $1.626(19)$ & $2.6295(9)$ & $173.3(17)$ \\
$\mathrm{C} 8-\mathrm{H} 8 A \cdots \mathrm{O} 3^{\text {ii }}$ & 0.98 & 2.56 & $3.4016(11)$ & 144 \\
\hline
\end{tabular}

Symmetry codes: (i) $-x,-y+2,-z+2$; (ii) $-x,-y+1,-z+2$.

centroid-to-centroid distance of 3.8018 (6) $\AA$ (symmetry codes: $x-1, y, z$ and $x+1, y, z)$ and slippage of $1.676 \AA$.

\section{Hirshfeld surface analysis}

The Hirshfeld surfaces mapped with normalized contact distance $d_{\text {norm }}$ and the two-dimensional fingerprint plots for I $\beta$ were generated using CrystalExplorer17.5 (Turner et al., 2017). The large and small red spots on the Hirshfeld surface mapped with $d_{\text {norm }}$ (Fig. 3) correspond to the $\mathrm{O} 2-\mathrm{H} 1 \mathrm{O} 2 \cdots \mathrm{O} 1$ and $\mathrm{C} 8-\mathrm{H} 8 \mathrm{~A} \cdots \mathrm{O} 3$ hydrogen bonds, respectively. The $\mathrm{H} \cdots \mathrm{O}$ distances are 1.09 and $0.16 \AA$ shorter than the sum of van der Waals radii of $\mathrm{H}$ and $\mathrm{O}$ atoms $(2.72 \AA)$. The $\mathrm{H} \cdots \mathrm{H}$ contact is the most populated contact and contributes $42.3 \%$ of the total intermolecular contacts, followed by $\mathrm{H} \cdots \mathrm{O} / \mathrm{O} \cdots \mathrm{H}(32.9 \%)$, $\mathrm{H} \cdots \mathrm{C} / \mathrm{C} \cdots \mathrm{H}(11.4 \%)$ and $\mathrm{C} \cdots \mathrm{C}(8.1 \%)$ contacts (Fig. 4). The tips of pseudo-mirrored sharp spikes at $d_{\mathrm{e}}+d_{\mathrm{i}} \simeq 1.6 \AA$ represent the shortest $\mathrm{H} \cdots \mathrm{O} / \mathrm{O} \cdots \mathrm{H}$ contacts, corresponding to the $\mathrm{O} 2-\mathrm{H} 1 O 2 \cdots \mathrm{O} 1$ hydrogen-bond. The absence of significant $\mathrm{C}-\mathrm{H} \cdots \pi$ interaction in the crystal structure of $\mathrm{I} \beta$ is indicated by the absence of characteristic 'wings' in the fingerprint plot of $\mathrm{H} \cdots \mathrm{C} / \mathrm{C} \cdots \mathrm{H}$ contacts. The $\mathrm{C} \cdots \mathrm{C}$ contacts include the weak $\pi-\pi$ interaction, which appears as a unique 'triangle' focused at $d_{\mathrm{e}} \simeq d_{\mathrm{i}} \simeq 1.8 \AA$. The $\pi-\pi$ interaction is illustrated as a unique pattern of red and blue 'triangles' on the shape-index surface and a flat region on the curvedness surface of the phenyl ring (see supporting Figures S1 and S2).

\section{Lattice energy calculation}

The lattice energies of polymorphs $\mathrm{I} \alpha$ and $\mathrm{I} \beta$ were calculated using PIXEL software (Gavezzotti, 2003) at default settings. The calculated lattice energy of $\mathrm{I} \alpha\left(107.5 \mathrm{~kJ} \mathrm{~mol}^{-1}\right)$ is larger than that of $\mathrm{I} \beta\left(98.5 \mathrm{~kJ} \mathrm{~mol}^{-1}\right)$ and this comparison is in agreement with the report of Pereira Silva et al. (2015), in which $\mathrm{I} \alpha$ is more stable than $\mathrm{I} \beta$ under ambient conditions.

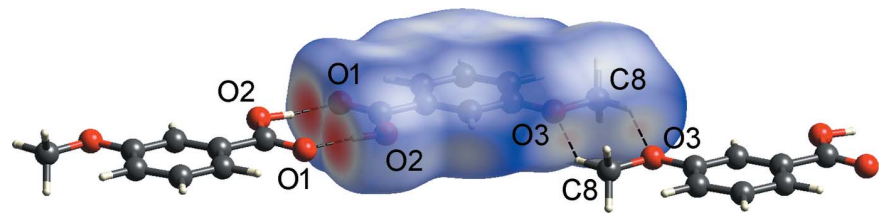

Figure 3

The Hirshfeld surface mapped over $d_{\text {norm }}$ of the central molecule of I $\beta$ hydrogen bonded to two neighbouring molecules. 

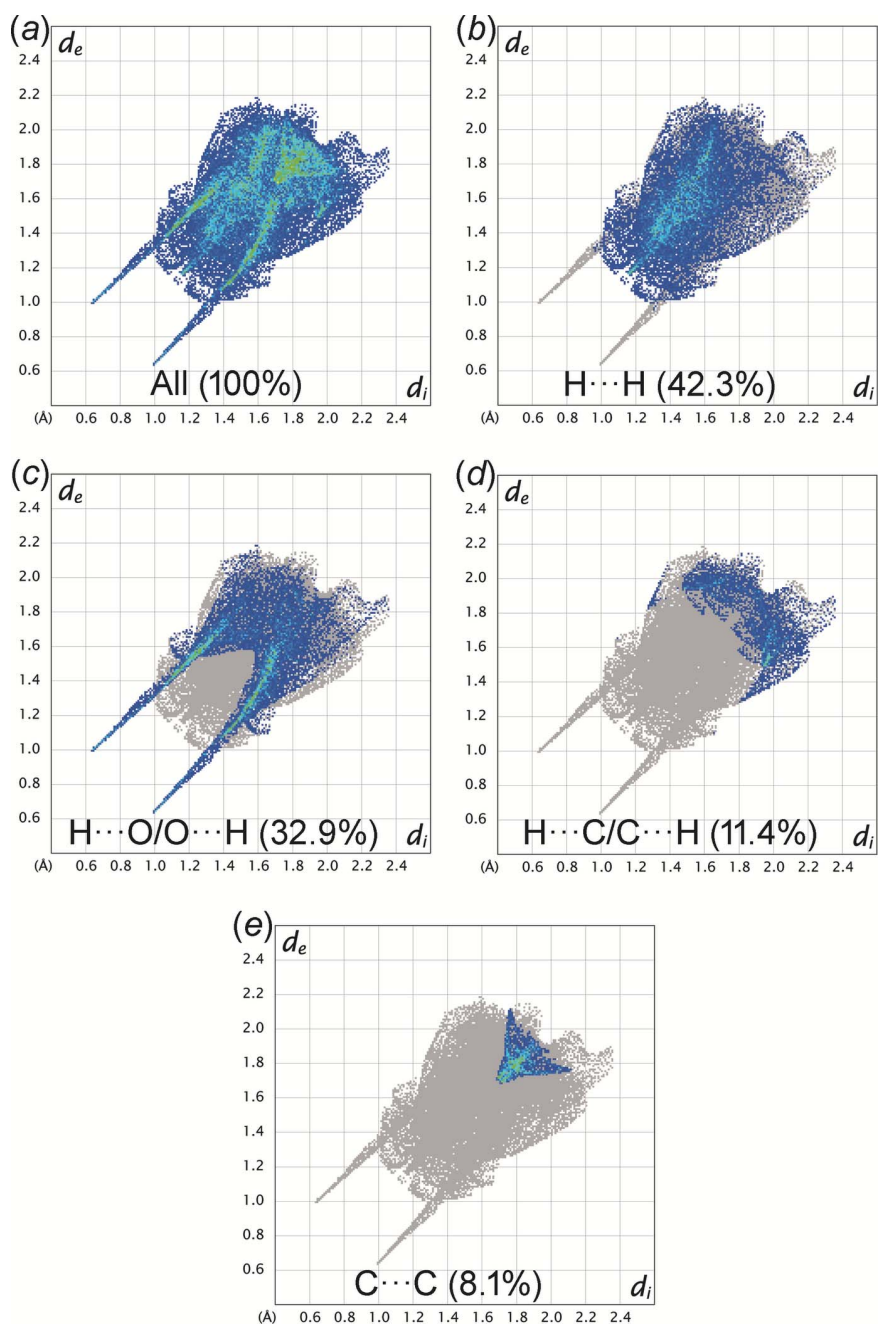

Figure 4

The two-dimensional fingerprint plots of $\mathrm{I} \beta$ for different intermolecular contacts giving their percentages of contribution to the Hirshfeld surface. $d_{\mathrm{i}}$ and $d_{\mathrm{e}}$ are the distances from the Hirshfeld surface to the nearest atom interior and exterior, respectively, to the surface.

\section{Database survey}

For the structure of 2-methoxybenzoic acid (refcodes FUFBOX and FUFBOX01, respectively), see: Parvez (1987) and Etter et al. (1988). For the structure of 4-methoxybenzoic acid (refcodes ANISIC, ANISIC01, ANISIC02 and ANISIC04, respectively), see: Bryan (1967), Colapietro \& Domenicano (1978), Fausto et al. (1997) and Hathwar et al. (2011). For the previous structure of 3-methoxybenzoic acid (refcodes EFINEO and EFINEO01, respectively), see: Raffo et al. (2014) and Pereira Silva et al. (2015).

\section{Synthesis and crystallization}

Single crystals of $\mathrm{I} \beta$ were obtained from an unsuccessful attempt of co-crystallization between 3-methoxybenzoic acid and hexamethylenetetramine. Colourless plate-like crystals were obtained from slow evaporation of a methanolic mixture
Table 2

Experimental details.

\begin{tabular}{|c|c|}
\hline \multicolumn{2}{|l|}{ Crystal data } \\
\hline Chemical formula & $\mathrm{C}_{8} \mathrm{H}_{8} \mathrm{O}_{3}$ \\
\hline$M_{\mathrm{r}}$ & 152.14 \\
\hline Crystal system, space group & Monoclinic, $P 2_{1} / c$ \\
\hline Temperature $(\mathrm{K})$ & 100 \\
\hline$a, b, c(\AA)$ & $\begin{array}{l}3.8018(4), 15.6027(16), \\
\quad 11.9755(12)\end{array}$ \\
\hline$\beta\left({ }^{\circ}\right)$ & $90.889(2)$ \\
\hline$V\left(\AA^{6}\right)$ & $710.28(13)$ \\
\hline$Z$ & 4 \\
\hline Radiation type & Mo $K \alpha$ \\
\hline$\mu\left(\mathrm{mm}^{-1}\right)$ & 0.11 \\
\hline Crystal size $(\mathrm{mm})$ & $0.56 \times 0.22 \times 0.12$ \\
\hline \multicolumn{2}{|l|}{ Data collection } \\
\hline Diffractometer & $\begin{array}{l}\text { Bruker SMART APEXII DUO } \\
\text { CCD }\end{array}$ \\
\hline Absorption correction & $\begin{array}{l}\text { Multi-scan (SADABS; Bruker, } \\
\text { 2009) }\end{array}$ \\
\hline$T_{\min }, T_{\max }$ & $0.881,0.987$ \\
\hline $\begin{array}{l}\text { No. of measured, independent and } \\
\text { observed }[I>2 \sigma(I)] \text { reflections }\end{array}$ & $9395,2550,2049$ \\
\hline$R_{\text {int }}$ & 0.023 \\
\hline$(\sin \theta / \lambda)_{\max }\left(\AA^{-1}\right)$ & 0.758 \\
\hline \multicolumn{2}{|l|}{ Refinement } \\
\hline$R\left[F^{2}>2 \sigma\left(F^{2}\right)\right], w R\left(F^{2}\right), S$ & $0.038,0.113,1.04$ \\
\hline No. of reflections & 2550 \\
\hline No. of parameters & 105 \\
\hline $\mathrm{H}$-atom treatment & $\begin{array}{l}\mathrm{H} \text { atoms treated by a mixture of } \\
\text { independent and constrained } \\
\text { refinement }\end{array}$ \\
\hline$\Delta \rho_{\max }, \Delta \rho_{\min }\left(\mathrm{e} \AA^{-3}\right)$ & $0.40,-0.27$ \\
\hline
\end{tabular}

Computer programs: APEX2 and SAINT (Bruker, 2009), SHELXS2013 and SHELXTL (Sheldrick, 2008), SHELXL2014 (Sheldrick, 2015), PLATON (Spek, 2009) and publCIF (Westrip, 2010).

of 3-methoxybenzoic acid and hexamethylenetetramine in equimolar ratio at room temperature.

\section{Refinement}

Crystal data, data collection and structure refinement details are summarized in Table 2. The $\mathrm{O}$-bound $\mathrm{H}$ atom was located from the difference-Fourier map and refined freely [O2$\mathrm{H} 1 O 2=1.01$ (2) $\AA$ ] . The remaining $\mathrm{H}$ atoms were positioned geometrically $[\mathrm{C}-\mathrm{H}=0.95$ and $0.98 \AA$ ] and refined using a riding model with $U_{\text {iso }}(\mathrm{H})=1.2$ or $1.5 U_{\text {eq }}(\mathrm{C})$. A rotating group model (AFIX 137) was applied to the methyl group.

\section{Funding information}

QAW thanks the Malaysian Government and USM for the award of the post of Research Officer under the Research University Individual Grant (1001/PFIZIK/8011080). HCK thanks the Malaysian Government for a MyBrain15 scholarship.

\section{References}

Bruker (2009). SADABS, APEX2 and SAINT. Bruker AXS Inc., Madison, Wisconsin, USA.

Bryan, R. F. (1967). J. Chem. Soc. B, pp. 1311-1316.

Colapietro, M. \& Domenicano, A. (1978). Acta Cryst. B34, 32773280. 
Etter, M. C., Urbańczyk-Lipkowska, Z., Fish, P. A., Panunto, T. W., Baures, P. W. \& Frye, J. S. (1988). J. Crystallogr. Spectrosc. Res. 18, 311-325.

Fausto, R., Matos-Beja, A. \& Paixão, J. A. (1997). J. Mol. Struct. 435, 207-218.

Gavezzotti, A. (2003). J. Phys. Chem. B, 107, 2344-2353.

Groom, C. R., Bruno, I. J., Lightfoot, M. P. \& Ward, S. C. (2016). Acta Cryst. B72, 171-179.

Hathwar, V. R., Thakur, T. S., Row, T. N. G. \& Desiraju, G. R. (2011). Cryst. Growth Des. 11, 616-623.

Parvez, M. (1987). Acta Cryst. C43, 2243-2245.
Pereira Silva, P. S., Castro, R. A. E., Melro, E., Silva, M. R., Maria, T. M. R., Canotilho, J. \& Eusébio, M. E. S. (2015). J. Therm. Anal. Calorim. 120, 667-677.

Raffo, P. A., Rossi, L., Alborés, P., Baggio, R. F. \& Cukiernik, F. D. (2014). J. Mol. Struct. 1070, 86-93.

Sheldrick, G. M. (2008). Acta Cryst. A64, 112-122.

Sheldrick, G. M. (2015). Acta Cryst. C71, 3-8.

Spek, A. L. (2009). Acta Cryst. D65, 148-155.

Turner, M. J., McKinnon, J. J., Wolff, S. K., Grimwood, D. J., Spackman, P. R., Jayatilaka, D. \& Spackman, M. A. (2017). CrystalExplorer17. University of Western Australia.

Westrip, S. P. (2010). J. Appl. Cryst. 43, 920-925. 


\section{supporting information}

Acta Cryst. (2019). E75, 8-11 [https://doi.org/10.1107/S2056989018016900]

Crystal structure of a second monoclinic polymorph of 3-methoxybenzoic acid with $Z^{\prime}=1$

Tze Shyang Chia, Huey Chong Kwong, Qin Ai Wong, Ching Kheng Quah and Md. Azharul Arafath

Computing details

Data collection: APEX2 (Bruker, 2009); cell refinement: SAINT (Bruker, 2009); data reduction: SAINT (Bruker, 2009); program(s) used to solve structure: SHELXS2013 (Sheldrick, 2008); program(s) used to refine structure: SHELXL2014 (Sheldrick, 2015); molecular graphics: SHELXTL (Sheldrick, 2008); software used to prepare material for publication: PLATON (Spek, 2009) and publCIF (Westrip, 2010).

3-Methoxybenzoic acid

Crystal data

$\mathrm{C}_{8} \mathrm{H}_{8} \mathrm{O}_{3}$

$M_{r}=152.14$

Monoclinic, $P 2_{1} / c$

$a=3.8018(4) \AA$

$b=15.6027(16) \AA$

$c=11.9755(12) \AA$

$\beta=90.889(2)^{\circ}$

$V=710.28(13) \AA^{3}$

$Z=4$

$F(000)=320$

$D_{\mathrm{x}}=1.423 \mathrm{Mg} \mathrm{m}^{-3}$

Mo $K \alpha$ radiation, $\lambda=0.71073 \AA$

Cell parameters from 3889 reflections

$\theta=2.6-32.4^{\circ}$

$\mu=0.11 \mathrm{~mm}^{-1}$

$T=100 \mathrm{~K}$

Plate, colourless

$0.56 \times 0.22 \times 0.12 \mathrm{~mm}$

\section{Data collection}

Bruker SMART APEXII DUO CCD diffractometer

Radiation source: fine-focus sealed tube Graphite monochromator $\varphi$ and $\omega$ scans

Absorption correction: multi-scan

(SADABS; Bruker, 2009)

$T_{\min }=0.881, T_{\max }=0.987$

9395 measured reflections

2550 independent reflections

2049 reflections with $I>2 \sigma(I)$

$R_{\text {int }}=0.023$

$\theta_{\text {max }}=32.6^{\circ}, \theta_{\min }=2.1^{\circ}$

$h=-5 \rightarrow 5$

$k=-21 \rightarrow 23$

$l=-18 \rightarrow 17$

\section{Refinement}

Refinement on $F^{2}$

Least-squares matrix: full

$R\left[F^{2}>2 \sigma\left(F^{2}\right)\right]=0.038$

$w R\left(F^{2}\right)=0.113$

$S=1.04$

2550 reflections

105 parameters

0 restraints

Primary atom site location: structure-invariant direct methods

Hydrogen site location: mixed

$\mathrm{H}$ atoms treated by a mixture of independent and constrained refinement

$w=1 /\left[\sigma^{2}\left(F_{\mathrm{o}}^{2}\right)+(0.0616 P)^{2}+0.1229 P\right]$

where $P=\left(F_{\mathrm{o}}^{2}+2 F_{\mathrm{c}}{ }^{2}\right) / 3$

$(\Delta / \sigma)_{\max }=0.001$ 
$\Delta \rho_{\max }=0.40$ e $\AA^{-3}$

$\Delta \rho_{\min }=-0.27 \mathrm{e} \AA^{-3}$

Special details

Geometry. All esds (except the esd in the dihedral angle between two 1.s. planes) are estimated using the full covariance matrix. The cell esds are taken into account individually in the estimation of esds in distances, angles and torsion angles; correlations between esds in cell parameters are only used when they are defined by crystal symmetry. An approximate (isotropic) treatment of cell esds is used for estimating esds involving l.s. planes.

Fractional atomic coordinates and isotropic or equivalent isotropic displacement parameters $\left(\hat{A}^{2}\right)$

\begin{tabular}{lllll}
\hline & $x$ & $y$ & $z$ & $U_{\text {iso }} * / U_{\mathrm{eq}}$ \\
\hline O1 & $0.20200(18)$ & $0.96657(4)$ & $0.88546(5)$ & $0.02247(16)$ \\
O2 & $-0.04494(18)$ & $0.88699(4)$ & $1.01876(5)$ & $0.02142(16)$ \\
$\mathrm{H} 1 \mathrm{O} 2$ & $-0.091(5)$ & $0.9451(12)$ & $1.0523(16)$ & $0.068(5)^{*}$ \\
O3 & $0.17686(17)$ & $0.58676(4)$ & $0.92170(5)$ & $0.01959(15)$ \\
$\mathrm{C} 1$ & $0.2204(2)$ & $0.81461(6)$ & $0.86862(7)$ & $0.01512(16)$ \\
$\mathrm{C} 2$ & $0.1573(2)$ & $0.73618(6)$ & $0.91952(7)$ & $0.01515(16)$ \\
$\mathrm{H} 2 \mathrm{~A}$ & 0.0504 & 0.7341 & 0.9906 & $0.018^{*}$ \\
$\mathrm{C} 3$ & $0.2516(2)$ & $0.66069(5)$ & $0.86565(7)$ & $0.01517(16)$ \\
$\mathrm{C} 4$ & $0.4082(2)$ & $0.66360(6)$ & $0.76109(7)$ & $0.01698(17)$ \\
$\mathrm{H} 4 \mathrm{~A}$ & 0.4738 & 0.6121 & 0.7245 & $0.020^{*}$ \\
$\mathrm{C} 5$ & $0.4674(2)$ & $0.74261(6)$ & $0.71099(7)$ & $0.01847(18)$ \\
$\mathrm{H} 5 \mathrm{~A}$ & 0.5727 & 0.7447 & 0.6396 & $0.022^{*}$ \\
$\mathrm{C} 6$ & $0.3755(2)$ & $0.81821(6)$ & $0.76329(7)$ & $0.01759(17)$ \\
$\mathrm{H} 6 \mathrm{~A}$ & 0.4169 & 0.8718 & 0.7283 & $0.021^{*}$ \\
$\mathrm{C} 7$ & $0.1244(2)$ & $0.89573(6)$ & $0.92491(7)$ & $0.01636(17)$ \\
$\mathrm{C} 8$ & $0.2877(2)$ & $0.50822(6)$ & $0.87173(7)$ & $0.01985(18)$ \\
$\mathrm{H} 8 \mathrm{~A}$ & 0.2229 & 0.4601 & 0.9198 & $0.030^{*}$ \\
$\mathrm{H} 8 \mathrm{~B}$ & 0.5434 & 0.5091 & 0.8628 & $0.030^{*}$ \\
$\mathrm{H} 8 \mathrm{C}$ & 0.1726 & 0.5017 & 0.7984 & $0.030^{*}$ \\
& & & &
\end{tabular}

Atomic displacement parameters $\left(\AA^{2}\right)$

\begin{tabular}{lllllll}
\hline & $U^{11}$ & $U^{22}$ & $U^{33}$ & $U^{12}$ & $U^{13}$ & $U^{23}$ \\
\hline O1 & $0.0301(3)$ & $0.0151(3)$ & $0.0223(3)$ & $-0.0001(2)$ & $0.0063(2)$ & $0.0011(2)$ \\
O2 & $0.0285(3)$ & $0.0176(3)$ & $0.0184(3)$ & $0.0009(2)$ & $0.0077(2)$ & $-0.0004(2)$ \\
O3 & $0.0254(3)$ & $0.0134(3)$ & $0.0202(3)$ & $0.0012(2)$ & $0.0073(2)$ & $0.0000(2)$ \\
C1 & $0.0147(3)$ & $0.0151(4)$ & $0.0156(3)$ & $0.0003(3)$ & $0.0006(2)$ & $-0.0005(3)$ \\
C2 & $0.0151(3)$ & $0.0165(4)$ & $0.0139(3)$ & $0.0002(3)$ & $0.0018(2)$ & $-0.0004(3)$ \\
C3 & $0.0146(3)$ & $0.0150(4)$ & $0.0159(3)$ & $0.0000(3)$ & $0.0013(2)$ & $0.0001(3)$ \\
C4 & $0.0161(3)$ & $0.0190(4)$ & $0.0160(3)$ & $0.0008(3)$ & $0.0025(3)$ & $-0.0026(3)$ \\
C5 & $0.0175(4)$ & $0.0231(4)$ & $0.0149(4)$ & $-0.0005(3)$ & $0.0035(3)$ & $-0.0001(3)$ \\
C6 & $0.0182(4)$ & $0.0183(4)$ & $0.0164(4)$ & $-0.0009(3)$ & $0.0023(3)$ & $0.0021(3)$ \\
C7 & $0.0164(3)$ & $0.0170(4)$ & $0.0157(3)$ & $0.0006(3)$ & $0.0008(3)$ & $0.0001(3)$ \\
C8 & $0.0217(4)$ & $0.0141(4)$ & $0.0238(4)$ & $0.0017(3)$ & $0.0033(3)$ & $-0.0029(3)$ \\
& & & & &
\end{tabular}


Geometric parameters $\left(\AA,{ }^{\circ}\right)$

\begin{tabular}{llll}
\hline $\mathrm{O} 1-\mathrm{C} 7$ & $1.2394(10)$ & $\mathrm{C} 3-\mathrm{C} 4$ & $1.3956(12)$ \\
$\mathrm{O} 2-\mathrm{C} 7$ & $1.3110(10)$ & $\mathrm{C} 4-\mathrm{C} 5$ & $1.3909(12)$ \\
$\mathrm{O} 2-\mathrm{H} 1 \mathrm{O} 2$ & $1.01(2)$ & $\mathrm{C} 4-\mathrm{H} 4 \mathrm{~A}$ & 0.9500 \\
$\mathrm{O} 3-\mathrm{C} 3$ & $1.3666(10)$ & $\mathrm{C} 5-\mathrm{C} 6$ & $1.3829(12)$ \\
$\mathrm{O} 3-\mathrm{C} 8$ & $1.4301(10)$ & $\mathrm{C} 5-\mathrm{H} 5 \mathrm{~A}$ & 0.9500 \\
$\mathrm{C} 1-\mathrm{C} 2$ & $1.3896(12)$ & $\mathrm{C} 6-\mathrm{H} 6 \mathrm{~A}$ & 0.9500 \\
$\mathrm{C} 1-\mathrm{C} 6$ & $1.4019(11)$ & $\mathrm{C} 8-\mathrm{H} 8 \mathrm{~A}$ & 0.9800 \\
$\mathrm{C} 1-\mathrm{C} 7$ & $1.4823(12)$ & $\mathrm{C} 8-\mathrm{H} 8 \mathrm{~B}$ & 0.9800 \\
$\mathrm{C} 2-\mathrm{C} 3$ & $1.3925(12)$ & $\mathrm{C} 8-\mathrm{H} 8 \mathrm{C}$ & 0.9800 \\
$\mathrm{C} 2-\mathrm{H} 2 \mathrm{~A}$ & 0.9500 & & \\
& & & 119.4 \\
$\mathrm{C} 7-\mathrm{O} 2-\mathrm{H} 1 \mathrm{O} 2$ & $109.8(11)$ & $\mathrm{C} 6-\mathrm{C} 5-\mathrm{H} 5 \mathrm{~A}$ & 119.4 \\
$\mathrm{C} 3-\mathrm{O} 3-\mathrm{C} 8$ & $116.94(7)$ & $\mathrm{C} 4-\mathrm{C} 5-\mathrm{H} 5 \mathrm{~A}$ & $119.09(8)$ \\
$\mathrm{C} 2-\mathrm{C} 1-\mathrm{C} 6$ & $120.52(8)$ & $\mathrm{C} 5-\mathrm{C} 6-\mathrm{C} 1$ & 120.5 \\
$\mathrm{C} 2-\mathrm{C} 1-\mathrm{C} 7$ & $120.47(7)$ & $\mathrm{C} 5-\mathrm{C} 6-\mathrm{H} 6 \mathrm{~A}$ & 120.5 \\
$\mathrm{C} 6-\mathrm{C} 1-\mathrm{C} 7$ & $119.01(8)$ & $\mathrm{C} 1-\mathrm{C} 6-\mathrm{H} 6 \mathrm{~A}$ & $122.85(8)$ \\
$\mathrm{C} 1-\mathrm{C} 2-\mathrm{C} 3$ & $119.62(7)$ & $\mathrm{O} 1-\mathrm{C} 7-\mathrm{O} 2$ & $121.76(7)$ \\
$\mathrm{C} 1-\mathrm{C} 2-\mathrm{H} 2 \mathrm{~A}$ & 120.2 & $\mathrm{O} 1-\mathrm{C} 7-\mathrm{C} 1$ & $115.39(7)$ \\
$\mathrm{C} 3-\mathrm{C} 2-\mathrm{H} 2 \mathrm{~A}$ & 120.2 & $\mathrm{O} 2-\mathrm{C} 7-\mathrm{C} 1$ & 109.5 \\
$\mathrm{O} 3-\mathrm{C} 3-\mathrm{C} 2$ & $115.43(7)$ & $\mathrm{O} 3-\mathrm{C} 8-\mathrm{H} 8 \mathrm{~A}$ & 109.5 \\
$\mathrm{O} 3-\mathrm{C} 3-\mathrm{C} 4$ & $124.26(7)$ & $\mathrm{O} 3-\mathrm{C} 8-\mathrm{H} 8 \mathrm{~B}$ & 109.5 \\
$\mathrm{C} 2-\mathrm{C} 3-\mathrm{C} 4$ & $120.31(8)$ & $\mathrm{H} 8 \mathrm{~A}-\mathrm{C} 8-\mathrm{H} 8 \mathrm{~B}$ & 109.5 \\
$\mathrm{C} 5-\mathrm{C} 4-\mathrm{C} 3$ & $119.34(8)$ & $\mathrm{O} 3-\mathrm{C} 8-\mathrm{H} 8 \mathrm{C}$ & 109.5 \\
$\mathrm{C} 5-\mathrm{C} 4-\mathrm{H} 4 \mathrm{~A}$ & 120.3 & $\mathrm{H} 8 \mathrm{~A}-\mathrm{C} 8-\mathrm{H} 8 \mathrm{C}$ & 109.5 \\
$\mathrm{C} 3-\mathrm{C} 4-\mathrm{H} 4 \mathrm{~A}$ & 120.3 & $\mathrm{H} 8 \mathrm{~B}-\mathrm{C} 8-\mathrm{H} 8 \mathrm{C}$ & \\
$\mathrm{C} 6-\mathrm{C} 5-\mathrm{C} 4$ & $121.13(8)$ & &
\end{tabular}

Hydrogen-bond geometry $\left(\AA,{ }^{\circ}\right)$

\begin{tabular}{lllll}
\hline$D-\mathrm{H} \cdots A$ & $D-\mathrm{H}$ & $\mathrm{H} \cdots A$ & $D \cdots A$ & $D-\mathrm{H} \cdots A$ \\
\hline $\mathrm{O} 2-\mathrm{H} 1 O 2 \cdots \mathrm{O} 1^{\mathrm{i}}$ & $1.008(19)$ & $1.626(19)$ & $2.6295(9)$ & $173.3(17)$ \\
$\mathrm{C} 8-\mathrm{H} 8 A \cdots \mathrm{O} 3^{\mathrm{ii}}$ & 0.98 & 2.56 & $3.4016(11)$ & 144 \\
\hline
\end{tabular}

Symmetry codes: (i) $-x,-y+2,-z+2$; (ii) $-x,-y+1,-z+2$. 